\title{
Wavelet and Mathematical Morphology as the De- Noising Methods for PD Analysis of High Voltage Transformer Windings
}

\author{
N H Nik Ali, M.S. Abd Rahman, J.A.Hunter, \\ P L Lewin \\ The Tony Davies High Voltage Laboratory \\ University of Southampton, United Kingdom \\ nhna1g13@soton.ac.uk
}

\author{
P Rapisarda \\ Communications, Signal Processing and Control \\ University of Southampton \\ Southampton, United Kingdom
}

\begin{abstract}
Partial discharge (PD) analysis is one of the most important techniques to evaluate the condition of the insulation systems within high voltage (HV) transformers. However, in typical field environments, measurements of PD signals can be distorted by noise sources. This greatly reduces the ability to identify PD sources in HV transformer windings. Therefore, denoising methods in PD analysis are very important. In recent years, several noise reduction techniques have been proposed for application in PD analysis. The common types of discharge events that may occur within high voltage transformer windings namely void, surface, corona and floating discharge have been experimentally generated. Each type of discharge was injected into different locations along a $\mathrm{HV}$ transformer winding and then measured using two wideband radio frequency current transformers (RFCTs) positioned at each end of the winding. Then, either the Discrete Wavelet Transform (DWT) and Stationary Wavelet Transform (SWT) or Mathematical Morphology (MM) were applied to reduce the noise in the raw captured PD signals. This paper presents the comparison of performance of the techniques in terms of noise reduction for this type of application.
\end{abstract}

Keywords-Partial discharge; de-noising; transformer windings

\section{INTRODUCTION}

Condition monitoring of $\mathrm{HV}$ electrical equipment, such as power transformers, using on-line PD measurement systems benefits both manufacturers and operators in many ways. For instance, obtaining information of the health of equipment, allows estimation and extension of the remaining service life of the equipment as well as planning of maintenance schedules [1] [2]. However, in a typical field environment, measured PD signals from the system are often distorted by noise sources and consequently may cause difficulties in PD analysis. Therefore, de-noising methods in PD analysis are very important. In recent years, several noise reduction techniques have been proposed for application in PD analysis such as low pass filtering, fast Fourier transforms, Wavelet transforms and hard thresholding [3]. In this paper, WT and MM [4] were used as the de-noising methods for PD analysis of HV transformer windings.
Previous work [5], in Southampton has developed nonlinear based techniques that facilitate the location of a PD source within an interleaved winding based on a method that generates two clusters in 3D space representing detected signals from each of the sensors. This paper is concerned with the effect of de-noising on the separation distance between the clusters. The performance of various techniques in term of noise reduction for this type of application are presented.

\section{EXPERIMENT}

The experiment consists of a HV transformer winding section, a $60 \mathrm{kV}$ transformer bushing, measurement sensors, PD source and an oscilloscope as shown in Fig. 1. The details of the measurement system are explained in [1]. The common types of discharge events that may occur within high voltage transformer windings are namely void, surface, corona and floating discharge have been experimentally generated [5] and then injected into different locations along the $\mathrm{HV}$ transformer windings with PD measurement using two wideband radio frequency current transformers (RFCTs) placed at each end of the winding. The WT and MM were used as de-noising methods to reduce the noise in the raw captured PD signals prior to processing.

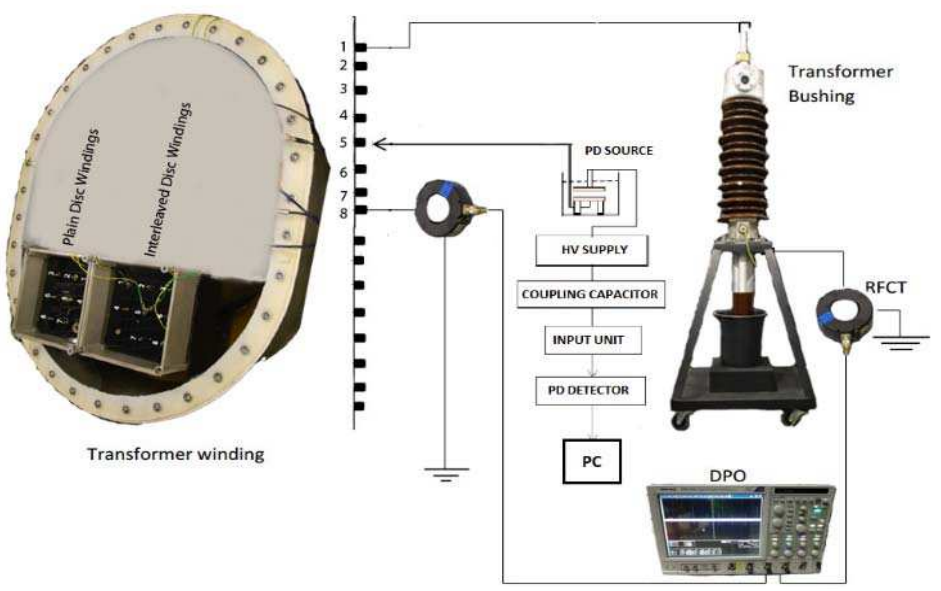

Fig.1. Experiment Setup 
For output signal decomposition, Wavelet analysis was used to decompose the original signals into nine detail levels and an approximation; these are presented in the form of ten element vectors. Then, in order to reduce the number of the element vectors obtained from wavelet analysis to three dimensions, Principle Component Analysis has been applied by using the three lower order of the principal components [1] [6].

\section{WAVELET TRANSFORM (WT) DE-NOISING}

The WT works like a pair of complementary high-pass (HPF) and low-pass filters (LPF), which divide up the original signal (S), into a series of approximation coefficients (cA1) and detail coefficients (cD1) at the $1^{\text {st }}$ level. The process is repeated iteratively with each iteration producing another approximation (cA) and detail (cD) coefficients. In term of denoising application, with proper thresholding in each level, coefficients that are estimated to be noise, can be removed from decomposed data and then a de-noised signal can be constructed. Basically, the wavelet de-noising procedure involves three steps: decomposition, thresholding and reconstruction.

In DWT, the outputs after each level of filtering were down sampled by a factor of two. Fig. 2 shows the process of the down sampling after the first level of filters.

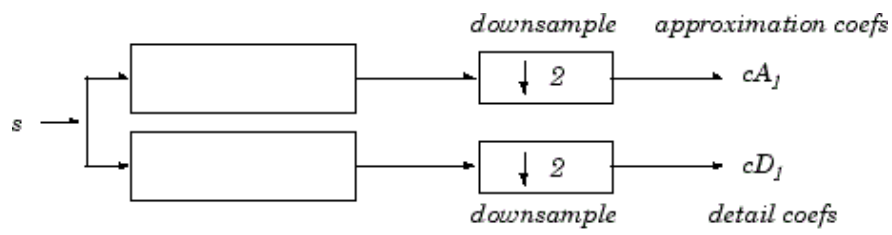

Fig. 2. Sampling down of discrete wavelet transform

The difference between SWT and DWT is the problem of down sampling after each level of filtering in DWT are eliminated in the SWT. In SWT, the low pass and high pass filters at each level of decomposition up sampled by putting a zero between each of the filter's coefficients from the previous level of decomposition. Therefore, the number of coefficients at each level of decomposition is as long as the original signal.

\section{MATHEMATICAL MORPHOLOGY (MM) DE-NOISING}

Although the WT is considered as a very good noise reduction method, it is still accompanied with some interference and distortion [7]. In this paper, MM has also been implemented to compare the performance of noise reduction of both approaches.

The operation of MM is based on mathematical operators which are applied on the captured PD signal using structure elements (SE). A structure element is a predefined shape used to interact with a given signal, with the purpose of determining how this shape fits the shape of the signal. According to [8], the shape of the SE will have only a small effect on the analysis.
There are two types of structure elements; flat structuring elements and non-flat structuring elements. In this paper, flat structuring elements are used due to simplicity since the only parameter which must be selected in this application is its length. Furthermore, MM operations using flat structuring elements are reported to be good for detecting peaks of the signal [9], for example identifying the positive and negative peaks of any captured PD signal.

The operators of morphology consist of dilation, erosion, opening and closing. Each of the operators will give different extracted shapes. The dilation operator enlarges positive peaks while the erosion operator minimizes positive peaks. On the other hand, the opening operator maintains negative peaks and cuts positive peaks while the closing operator maintains positive peaks and cuts negative peaks [10]. Among the four operators, average opening and closing operators were used in this application as they preserve negative and positive peaks in the data.

\section{COMPARISON CRITERIA}

\section{A. Compression Ratio (CR)}

$\mathrm{CR}$ calculates the ratio of the reconstructed signal to the original signal and this ratio is defined as:

$$
C R=\frac{E_{r}}{E_{o}} \times 100 \%
$$

where $E_{r}$ and $E_{o}$ are the total energy of the reconstructed signal and original signal respectively. The energy, E can be calculated using Parseval's theorem which is defined as:

$$
E=\sum_{i=1}^{N}\left|S_{i}\right|^{2}
$$

where $\mathrm{S}$ and $\mathrm{N}$ are the pulse magnitude and number of captured PD signals.

\section{B. Mean Square Error (MSE)}

MSE was used to compare the effectiveness of the denoising methods where:

$$
M S E=\frac{1}{N} \sum_{i=1}^{N}(S(i)-R(i))^{2},
$$

where $\mathrm{S}(\mathrm{i})$ and $\mathrm{R}(\mathrm{i})$ represent original signal without noise and recovered signal after de-noising respectively and $\mathrm{N}$ is length of the signal.

\section{Peak Signal to Noise Ratio (PSNR)}

Another criteria that can be used to measure the reconstruction accuracy after de-noising is PSNR and is defined as:

$$
P S N R=20 \log _{10}\left(\frac{A_{\max }}{M S E}\right),
$$

where $A_{\max }$ is the maximum amplitude of the signal. 


\section{Separation Distance}

Previous research [5] has shown that the measurement data obtained from the RFCTs at two measurement points either end of a winding can be clustered in 3-D space using principle components. In this paper, following the de-noising process, the separation length between the centroid of clusters produced has been determined.

The centroid of clusters can be calculated by taking the average of all points in the clusters using:

$$
\begin{aligned}
C_{\mathrm{x}} & =1 / \mathrm{N}_{\mathrm{p}}\left(\sum_{\mathrm{i}=1}^{\mathrm{N}} \mathrm{x}_{\mathrm{i}}\right), \\
C_{y} & =1 / N_{p}\left(\sum_{i=1}^{N} y_{i}\right), \\
C_{z} & =1 / N_{p}\left(\sum_{i=1}^{N} z_{i}\right),
\end{aligned}
$$

where $\mathrm{x}, \mathrm{y}$ and $\mathrm{z}$ represent first, second and third principle component while $\mathrm{N}_{\mathrm{p}}$ is the number of points in clusters. The magnitude, $\mathrm{P}$ with respect to each principle component and separation distance, $\mathrm{D}(\mathrm{x}, \mathrm{y}, \mathrm{z})$ between them in three dimensions is calculated using:

$$
\begin{array}{r}
P x_{12}=\left(C x_{1}-C x_{2}\right)^{2}, \\
P y_{12}=\left(C y_{1}-C y_{2}\right)^{2}, \\
P z_{12}=\left(C z_{1}-C z_{2}\right)^{2}, \\
D(x, y, z)=\sqrt{\left(P x_{12}+P y_{12}+P z_{12}\right)^{2}},
\end{array}
$$

where 1 and 2 represent the first cluster and second cluster in the 3-D space of the principle component.

\section{RESULTS AND DISCUSSION}

\section{A. Performance of The De-noising techniques}

In WT, for both methods, the mother wavelet of $\mathrm{db} 9$ and level 1 of decomposition while for MM, length 2 of structure element was used. Table 1 to Table 3 show the results for the comparison criteria applied for all types of discharge at all terminals.

Table 1. Comparison criteria of de-noising using DWT.

\begin{tabular}{|c|c|c|c|}
\hline Criteria & $\begin{array}{c}\text { Average } \\
\text { CR }\end{array}$ & $\begin{array}{c}\text { Average } \\
\text { MSE }\end{array}$ & $\begin{array}{c}\text { Average } \\
\text { PSNR }\end{array}$ \\
\hline Void Signal & 99.0801 & $2.5377 \times 10^{-7}$ & 114.7482 \\
\hline Corona Signal & 98.8354 & $2.0387 \times 10^{-7}$ & 115.8379 \\
\hline Surface Signal & 99.3911 & $1.2792 \times 10^{-7}$ & 117.2453 \\
\hline Floating Signal & 99.1602 & $1.901 \times 10^{-6}$ & 109.2937 \\
\hline
\end{tabular}

Table 2. Comparison criteria of de-noising using SWT.

\begin{tabular}{|c|c|c|c|}
\hline Criteria & $\begin{array}{c}\text { Average } \\
\text { CR }\end{array}$ & $\begin{array}{c}\text { Average } \\
\text { MSE }\end{array}$ & $\begin{array}{c}\text { Average } \\
\text { PSNR }\end{array}$ \\
\hline Void Signal & 99.8065 & $1.3506 \times 10^{-7}$ & 117.1211 \\
\hline Corona Signal & 98.4865 & $1.2255 \times 10^{-7}$ & 117.7616 \\
\hline Surface Signal & 99.2096 & $7.6009 \times 10^{-8}$ & 119.3428 \\
\hline Floating Signal & 98.9207 & $6.0499 \times 10^{-7}$ & 112.6378 \\
\hline
\end{tabular}

Table 3. Comparison criteria of de-noising using MM

\begin{tabular}{|c|c|c|c|}
\hline Criteria & $\begin{array}{c}\text { Average } \\
\text { CR }\end{array}$ & $\begin{array}{c}\text { Average } \\
\text { MSE }\end{array}$ & $\begin{array}{c}\text { Average } \\
\text { PSNR }\end{array}$ \\
\hline Void Signal & 98.7653 & $1.2871 \times 10^{-4}$ & 101.8449 \\
\hline Corona Signal & 98.4133 & $1.0985 \times 10^{-4}$ & 102.4563 \\
\hline Surface Signal & 99.1786 & $6.7223 \times 10^{-5}$ & 104.5834 \\
\hline Floating Signal & 98.7433 & $3.200 \times 10^{-3}$ & 92.6034 \\
\hline
\end{tabular}

Analysis of the tables reveals that SWT produced the best performance as it has higher PSNR indicating the reconstructed signal is the most similar to the original signal. In addition, it has a smaller value of MSE indicating that the reconstructed signal resembles the original signal better than results obtained using other methods.

\section{B. Effect of Length in $M M$}

As flat structuring was used, the length of the SE plays an important role in this application. Fig. 3 shows the effect of SE length of 2, 5, 10 and 15 on one of the captured signals.

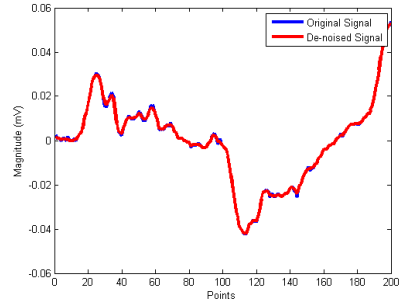

(a)

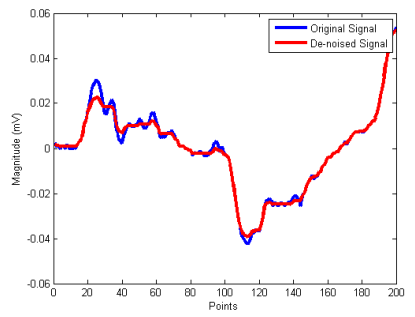

(c)

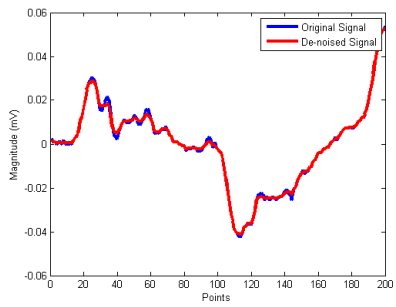

(b)

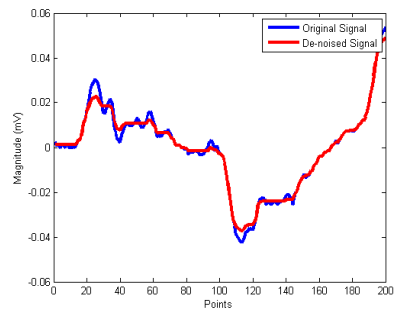

(d)
Fig. 3. Effect of SE length of (a) 2 (b) 5 (c) 10 (d) 15.

Increasing the length of SE, reduced the magnitude of the signals as shown in Fig. 3 and reduced the overall performance of this de-noising application. Table 4 shows the results of the effect of SE length in terms of compression ratio, mean square error and peak signal to noise ratio.

Table 4. Effect of SE length.

\begin{tabular}{|c|c|c|c|}
\hline $\begin{array}{c}\text { Criteria } \\
\text { SE length }\end{array}$ & $\begin{array}{c}\text { Average } \\
\text { CR }\end{array}$ & $\begin{array}{c}\text { Average } \\
\text { MSE }\end{array}$ & $\begin{array}{c}\text { Average } \\
\text { PSNR }\end{array}$ \\
\hline 1 & 100 & 0 & $\infty$ \\
\hline 2 & 98.7653 & $1.2871 \times 10^{-4}$ & 101.8449 \\
\hline 5 & 98.3305 & $1.2853 \times 10^{-4}$ & 100.8687 \\
\hline 10 & 97.2915 & $1.2752 \times 10^{-4}$ & 99.6567 \\
\hline 15 & 95.8663 & $1.2628 \times 10^{-4}$ & 99.0739 \\
\hline
\end{tabular}




\section{Separation Distance}

To determine the effect of de-noising in terms of the separation distance between the clusters, the best de-noising result which has the best PSNR was used and compared with the original separation distance between the clusters. Fig 4 shows an example of the plot of the clusters in 3-D space of principle component for a void discharge injected into terminal 1 of the transformer winding.

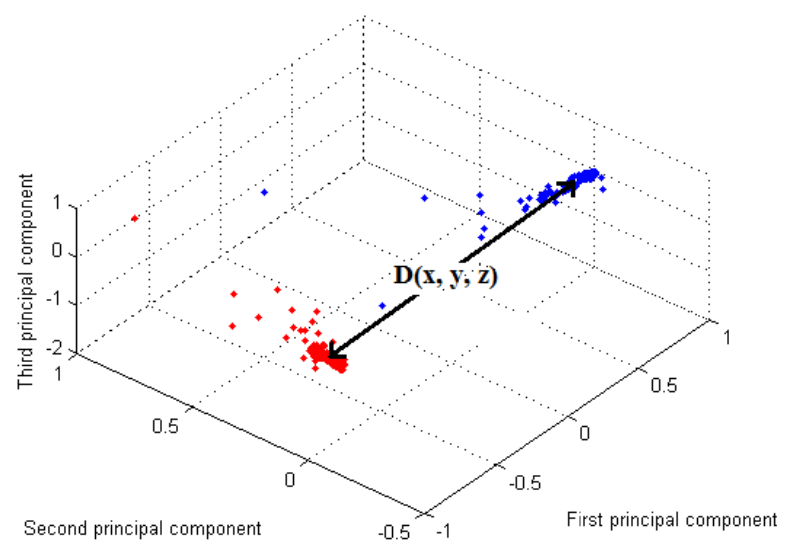

Fig. 4. Clusters in 3-D space of principle component

The distance, $\mathrm{D}(\mathrm{x}, \mathrm{y}, \mathrm{z})$ between the centroid of the clusters was calculated between the clusters and Table 5 shows the results for different types of discharge at terminal 1. Table 6 shows the separation distance between centroids of the clusters for a void discharge at terminal 1- terminal 8.

Table 5. Separation distance between centroid of the clusters for all type of discharge at terminal 1.

\begin{tabular}{|c|c|c|c|}
\hline Signal & $\begin{array}{c}\text { Original } \\
\text { separation }\end{array}$ & $\begin{array}{c}\text { De-noised } \\
\text { separation }\end{array}$ & $\begin{array}{c}\text { Increment } \\
\text { Percentage } \\
\mathbf{( \% )}\end{array}$ \\
\hline Void Signal & 0.7046 & 0.7085 & 0.55 \\
\hline Corona Signal & 0.7999 & 0.8071 & 0.89 \\
\hline Surface Signal & 0.7289 & 0.7414 & 1.71 \\
\hline Floating Signal & 0.7594 & 0.7679 & 0.85 \\
\hline
\end{tabular}

Table 6. Separation distance between centroid of the clusters for void discharge at terminal 1- terminal 8 .

\begin{tabular}{|c|c|c|c|}
\hline Terminal & $\begin{array}{c}\text { Original } \\
\text { separation }\end{array}$ & $\begin{array}{c}\text { De-noised } \\
\text { separation }\end{array}$ & $\begin{array}{c}\text { Increment } \\
\text { Percentage } \\
(\mathbf{\%})\end{array}$ \\
\hline 1 & 0.7046 & 0.7085 & 0.55 \\
\hline 2 & 0.6956 & 0.6996 & 0.57 \\
\hline 3 & 0.6033 & 0.6075 & 0.69 \\
\hline 4 & 0.3656 & 0.3686 & 0.81 \\
\hline 5 & 0.1996 & 0.2013 & 0.84 \\
\hline 6 & 0.1550 & 0.1568 & 1.14 \\
\hline 7 & 0.3712 & 0.3736 & 0.64 \\
\hline 8 & 0.6086 & 0.6125 & 0.63 \\
\hline
\end{tabular}

The average percentage in increment of separation distance for all types of discharge at all terminals was $0.80 \%$. While the maximum and minimum percentage of the increment were $1.30 \%$ and $0.37 \%$ respectively.

\section{CONCLUSION}

The use of a de-noising algorithm in clustering applications for PD analysis gives an improvement in terms of separation distance between clusters produced in 3-D space using principle components. The results obtained here were generated under laboratory conditions; it is hypothesized that a greater improvement in performance will be seen when analyzing data captured in the field. This is currently being investigated.

\section{REFERENCES}

[1] N. H. Nik Ali, J. Hunter, P. Rapisarda and P. Lewin, "Identification of multiple partial discharge sources in high voltage transformer windings," in IEEE Conference on Electrical Insulation and Dielectric Phenomena (CEIDP), 2014, Des Moines, IA, 19-22 Oct. 2014.

[2] E. Gockenbach and H. Borsi, "Condition monitoring and diagnosis of power transformers," International Symposium on Electrical Insulating Materials (ISEIM), pp. 16-19, 2008.

[3] S. Sriram, S. Nitin, K. Prabhu and M. Bastiaans, "Signal denoising techniques for partial discharge measurements," IEEE Transactions on Dielectrics and Electrical Insulation, vol. 12, no. 6, pp. 1182 - 1191, 2006.

[4] J. Chan, H. Ma, T. Saha and C. Ekanayake, "Self-adaptive partial discharge signal de-noising based on ensemble empirical mode decomposition and automatic morphological thresholding," Dielectrics and Electrical Insulation, IEEE Transactions on, vol. 21, no. 1, pp. 294 - 303, 2014.

[5] M. S. A. Rahman, "Identification of Partial Discharge Sources and Their Location within High Voltage Transformer Windings," in Ph.D thesis, School Electronics and Computer Science, University of Southampton, 2014.

[6] L. Hao, P. Lewin, J. Hunter, D. Swaffield, A. Contin, C. Walton and M. Michel, "Discrimination of multiple PD sources using wavelet decomposition and principal component analysis," IEEE Transactions on Dielectrics and Electrical Insulation, vol. 18, no. 5, pp. 1702 1711, October 2011.

[7] Z. Chen, N. Gao and W. Sun, "A Signal Based Triangular Structuring Element for Mathematical Morphological Analysis and Its Application in Rolling Element Bearing Fault Diagnosis," in Shock and Vibration, vol. 2014, Article ID 590875, 2014.

[8] Y. Dong, M. Liao, X. Zhang and F. Wang, "Faults diagnosis of rolling element bearings based on modified morphology method," Mech. Syst. Signal Process, vol. 25, no. 4, pp. 1276 - 1286, 2011.

[9] N. Nikolaou and I. Antoniadis, "Application Of Morphological Operators As Envelope Extractors For Impulsive-Type Periodic Signals," Mechanical Systems and Signal Processing, vol. 17, no. 6, pp. 1147 - 1162, 2003.

[10] J. Chan, H. Ma and T. Saha, "Automatic Blind Equalization and Thresholding for Partial Discharge Measurement in Power Transformer," Power Delivery, IEEE Transactions on, vol. 29, no. 4, pp. 1927 - 1938, 2014. 